\title{
On some 1/4 BPS Wilson-'t Hooft loops
}

\section{Chang-Yong Liu and Li Qin}

College of Science, Northwest A\&F University, Yangling, Shaanxi 712100, China

E-mail: liuchangyong@nwsuaf.edu.cn, qinli2009@foxmail.com

ABSTRACT: In this paper, we investigate the 1/4 BPS Wilson-'t Hooft loops in $\mathcal{N}=4$ supersymmetric Yang-Mills theory. We use the bulk D3-brane solutions with both electric and magnetic charges on its world-volume to describe some of $1 / 4$ BPS Wilson-'t Hooft loops. The D3-brane supersymmetric solutions are derived form requiring $\kappa$-symmetry. We find the two consistent constraints for Killing spinors and calculate the conserved charges of straight 1/4 BPS Wilson-'t Hooft loops and expectation values of circular 1/4 BPS Wilson-'t Hooft loops separately.

KeYwords: Wilson, 't Hooft and Polyakov loops, D-branes, AdS-CFT Correspondence

ARXiv EPrint: 1505.06399 


\section{Contents}

1 Introduction 1

2 Wilson-'t Hooft loops with insertions 3

2.1 Review of the straight 1/4 BPS Wilson loops 3

2.2 D3-brane solution 5

2.3 Conserved charges 8

3 1/4 BPS circular Wilson-'t Hooft loop $\quad 9$

3.1 Review of the 1/4 BPS circular Wilson loop 9

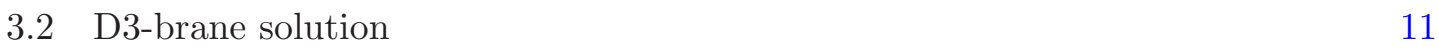

3.3 The 1/4 BPS Wilson-'t Hooft loop expectation value 13

$\begin{array}{lll}\text { 3.3.1 The expression of expectation value } & 13\end{array}$

$\begin{array}{lll}\text { 3.3.2 The physical meaning of } c & 15\end{array}$

$\begin{array}{ll}\text { 3.3.3 The } \mathrm{SL}(2, Z) \text { symmetry } & 16\end{array}$

4 Conclusions and discussions $\quad 16$

\section{Introduction}

Wilson loop operators are interesting observables in gauge field theories. These operators can be viewed as the worldlines of a very massive quark with the electric charge. In $\mathcal{N}=4$ SYM, the Wilson loops have the general form

$$
W(\mathcal{C})=\operatorname{Tr} \mathcal{P} \exp \left(i \int_{\mathcal{C}}\left(A_{\mu} \dot{x}^{\mu}+\Phi_{i} \theta^{i}|\dot{x}|\right) d s\right)
$$

where $x^{i}$ is the parametrization of the loop $\mathcal{C}$ and $\theta^{i}$ is a unit vector in $R^{6}$. The $A_{\mu}$ are the gauge fields and $\Phi_{i}$ are the six scalars in the adjoint representation.

According to the $A d S / C F T$ correspondence, the expectation values of fundamental representation Wilson loops are calculated on the AdS side as fundamental string action bounded by the curve $\mathcal{C}$ at the boundary $[1-7]$ :

$$
W(\mathcal{C})=\int_{\partial X=\mathcal{C}} \mathcal{D} X \exp (-\sqrt{\lambda} S[X]),
$$

where the $S[X]$ is the string action. For large $\lambda$, the expectation value of the Wilson loop is given by the area $A$ of the minimal surface bounded by $\mathcal{C}$ as

$$
W(\mathcal{C}) \sim \exp (-\sqrt{\lambda} A) .
$$


These fundamental strings sweep out a worldsheet in AdS side. There are two simple 1/2 BPS Wilson loops: the infinitely straight line and circular Wilson loop.

For the infinitely straight line Wilson loop, its expectation value is simple

$$
W_{\text {line }}=1 \text {. }
$$

The infinitely straight line Wilson loop preserves half of the supersymmetries. For the circular Wilson loop which also preserves half the supersymmetries, its expectation value is

$$
W_{\text {circle }}=\exp (\sqrt{\lambda})
$$

The circular Wilson loop can be calculated in perturbation theory by reducing the calculation of the rainbow/ladder diagrams to matrix model [8]

$$
W_{\text {circular }}=\frac{1}{Z} \int \mathcal{D} M \frac{1}{N} \operatorname{Tr} e^{M} e^{-\frac{2 N}{\lambda} \operatorname{Tr} M^{2}}
$$

The leading behavior at large $\mathrm{N}$, it is

$$
W_{\text {circular }} \sim \frac{2}{\sqrt{\lambda}} I_{1}(\sqrt{\lambda}) \sim e^{\sqrt{\lambda}}
$$

where $I_{1}(x)$ is the Bessel function.

For Wilson loops with high rank representation of gauge group, we must consider the interaction between strings. These stings can blow up to D3-branes and/or D5-branes configurations corresponding to symmetric and anti-symmetric representation separately [9-22] analogous to the gaint gravitons [23-26]. The expectation value of $1 / 2$ BPS circular Wilson loop in the symmetric representation described by D3-brane is

$$
W_{\mathrm{sym}}=e^{2 N\left(\kappa \sqrt{1+\kappa^{2}}+\operatorname{arcsinh} \kappa\right)}
$$

with $\kappa=\frac{k \sqrt{\lambda}}{4 N}$. The expectation value of BPS circular Wilson loop in the Anti-symmetric representation described by $\mathrm{D} 5$-brane is

$$
W_{\text {asym }}=e^{\frac{2 N \sqrt{\lambda}}{3 \pi} \sin ^{3} \theta_{k}},
$$

where $\theta_{k}$ is related to $k$ by

$$
k=\frac{2 N}{\pi}\left(\frac{1}{2} \theta_{k}-\frac{1}{4} \sin 2 \theta_{k}\right) .
$$

The $\mathcal{N}=4$ SYM and the type IIB string theory have the electric-magnetic duality $\mathrm{SL}(2, Z)$. Under S-duality, the electric charge is replaced with the magnetic charge. We need consider another important loops known as Wilson-'t Hooft loops. The Wilson-'t Hooft loops are the dyon worldlines wich carry both the electric and magnetic charges in gauge theory $[27,28]$. In purely electric case, they reduce to the Wilson loops. They are classified by a pair of weights (electric and magnetic) for the gauge group and its magnetic dual, modulo the action of the Weyl group. From the string theory, the Wilson-'t Hooft 
loops are the $(F 1, D 1)$ bound states ending on the worldvolume of D3-branes [29]. For a $(n, m)$-string, the string tension is

$$
\tau_{n, m}=\frac{\sqrt{n^{2}+m^{2} / g_{s}^{2}}}{2 \pi \alpha^{\prime}}
$$

which is invariant under $S$-duality: $\left(n, m, g_{s}, \alpha^{\prime}\right) \leftrightarrow\left(-m, n, g_{s}^{-1}, \alpha^{\prime} g_{s}\right)$. The $1 / 2$ BPS Wilson-'t Hooft loops have been discussed in [30]. The expectation value of $1 / 2$ circular BPS Wilson-'t Hooft loop is the same as (1.8) with

$$
\kappa^{2}=\frac{\pi}{4 N} \frac{|n+m \tau|^{2}}{\operatorname{Im} \tau}
$$

which is invariant under the $\mathrm{SL}(2, Z)$ transformation

$$
\begin{array}{cl}
\tau \rightarrow & \frac{a \tau+b}{c \tau+d} \\
a d-b c=1, \quad & a, b, c, d \in \mathbb{Z},
\end{array}
$$

where the Yang-Mills coupling $\tau$ is related to the string coupling as following

$$
\tau=\frac{4 \pi i}{g_{Y M}^{2}}+\frac{\theta}{2 \pi}=\frac{i}{g_{s}}+\frac{\chi}{2 \pi} .
$$

In this paper, we study the 1/4 BPS Wilson-'t Hooft loops using D-branes description. We consider 1/4 BPS Wilson-'t Hooft loops in symmetric representation. Similar to the $F 1$ 's, these can be studed by using the D3-branes configuration. The paper is organized as follows. In section 2, we study the Wilson-'t Hooft loops with insertions using D3-brane description and conserved charges. In section 3 , we discuss the $1 / 4$ BPS circular Wilson-'t Hooft loops. We end with the conclusions and discussions.

\section{Wilson-'t Hooft loops with insertions}

\subsection{Review of the straight 1/4 BPS Wilson loops}

The 1/4 BPS Wilson loops can be constructed by Wilson loops with two insertions [31]. These deformations of Wilson loop are related to a certain spin-chain systems and can be solved by Bethe ansatz. Without the local insertions, these supersymmetric Wilson loops preserve half the supersymmetries of the vacuum. Considering $1 / 2$ BPS local insertions, we can construct the the $1 / 4$ BPS Wilson loops on $R \times S^{3}$

$$
W_{Z^{J}}=\operatorname{Tr} P\left[Z^{J}(-\infty) e^{i \int_{-\infty}^{\infty}\left(A_{t}(t, 0)+\Phi_{3}(t, 0)\right) d t} \bar{Z}^{J}(\infty) e^{i \int_{\infty}^{-\infty}\left(A_{t}(t, \pi)+\Phi_{3}(t, \pi)\right) d t}\right] .
$$

Where $Z=\Phi_{1}+i \Phi_{2}$ is a half-BPS complex scalar. The angle 0 and $\pi$ are two points on $S^{3}$.

In the dual string theory on $A d S_{5} \times S^{5}$, the $1 / 4$ BPS Wilson loops in supergravity extend to the two lines on the boundary which one line run up and another line run down at antipodal point on the $R \times S^{3}$ boundary with one of the insertions to the infinite past and the other one to future infinity in global Lorentzian $A d S_{5}$. 
It is convenient to use the following global coordinates for $A d S_{5}$ :

$$
\frac{d s^{2}}{L^{2}}=-\cosh ^{2} \rho d t^{2}+d \rho^{2}+\sinh ^{2} \rho\left(d \chi^{2}+\sin ^{2} \chi\left(d \vartheta^{2}+\sin ^{2} \vartheta d \varphi^{2}\right)\right)+d \theta^{2}+\sin ^{2} \theta d \phi^{2} .
$$

Where $L^{4}=\lambda \alpha^{\prime 2}$ is the radius of $A d S_{5}$ and $S^{5}$. The Green-Schwarz string action is

$$
\mathcal{S}=\frac{L^{2}}{4 \pi \alpha^{\prime}} \int d \sigma d \tau \sqrt{-h} h^{\alpha \beta}\left[-\cosh ^{2} \rho \partial_{\alpha} t \partial_{\beta} t+\partial_{\alpha} \rho \partial_{\beta} \rho+\partial_{\alpha} \theta \partial_{\beta} \theta+\sin ^{2} \theta \partial_{\alpha} \phi \partial_{\beta} \phi\right] .
$$

Adopting a periodic ansatz

$$
\rho=\rho(\sigma), \quad t=\omega \tau, \quad \theta=\theta(\sigma), \quad \phi=\omega_{1} \tau,
$$

the equations of motion are

$$
\begin{aligned}
\rho^{\prime \prime}-\omega^{2} \cosh \rho \sinh \rho & =0, \\
\theta^{\prime \prime}+\omega_{1}^{2} \cos \theta \sin \theta & =0 .
\end{aligned}
$$

The solution to the string equations of motion is

$$
\phi=t, \quad \sin \theta=\frac{1}{\cosh \rho} .
$$

This solution satisfies the correct boundary condition. The surface approaches the boundary of $A d S_{5}$ and get to the north-pole of $S^{2}$ associated to the scalar $\Phi_{3}$ at $\sigma=0$. As $\sigma \rightarrow \infty$, the string comes close to the center of $A d S_{5}$ and rotates around the equator of $S^{2}$ carrying the angular momentum related to $Z^{J}$. There are two parts to the sting: at $\chi=0$ and at $\chi=\pi$. They are continuously connected to each other beyond $\rho=0$.

Using the supersymmetry analysis, the string solution preserves $1 / 4$ of the supersymmetries. The $\kappa$-symmetry projector associated with a fundamental string is

$$
\Gamma=\frac{1}{\sqrt{-\operatorname{det} g}} \partial_{t} x^{\mu} \partial_{\rho} x^{\nu} \gamma_{\mu} \gamma_{\nu} K
$$

Where $\mathrm{K}$ acts on spinors by complex conjugation. $\gamma_{\mu}=e_{\mu}^{a} \Gamma_{a}$ and $\Gamma_{a}$ are constant tangent space gamma-matrices. $\Gamma_{\star}=\Gamma^{0} \Gamma^{1} \Gamma^{2} \Gamma^{3} \Gamma^{4}$ is the product of all gamma matrices in the $A d S_{5}$ direction. The number of supersymmetries preserved by the string is the number of the independent solutions to the equation $\Gamma \epsilon=\epsilon$. The dependence of the Killing spinors on the relevant coordinates is written as

$$
\epsilon=e^{-\frac{i}{2} \rho \Gamma_{\star} \Gamma_{1}} e^{-\frac{i}{2} t \Gamma_{\star} \Gamma_{0}} e^{-\frac{i}{2} \theta \Gamma_{\star} \Gamma_{5}} e^{\frac{1}{2} \phi \Gamma_{56}} \epsilon_{0} .
$$

Where $\epsilon_{0}$ is a constant chiral complex 16-component spinor. This satisfies the Killing spinor equation

$$
\left(\partial_{\mu}+\frac{1}{4} \omega_{\mu}^{a b} \Gamma_{a b}+\frac{i}{2 L} \Gamma_{\star} \gamma_{\mu}\right) \epsilon=0
$$

Using the vielbeins

$$
e^{0}=L \cosh \rho d t, \quad e^{1}=L d \rho, \quad e^{5}=L d \theta, \quad e^{6}=L \sin \theta d \phi
$$


and inserting the string solution (2.5) into the expression (2.6), it is easy to obtain two consistent condition

$$
\Gamma_{\star} \Gamma_{056} \epsilon_{0}=i \epsilon_{0}, \quad \Gamma_{01} K \epsilon_{0}=-\epsilon_{0} .
$$

Thus the string solution preserves $1 / 4$ of the supersymmetries. The total angular momentum carried by the string is given

$$
J=\int P_{\phi}=\frac{L^{2}}{\pi \alpha^{\prime}} \int_{0}^{\sigma_{\max }} d \sigma \sin ^{2} \theta \dot{\phi} .
$$

After concrete calculation, the energy carried by the string is

$$
E=\int P_{t}=\frac{L^{2}}{\pi \alpha^{\prime}} \int_{0}^{\sigma_{\max }} d \sigma \cosh ^{2} \rho \dot{t}=J
$$

These solutions satisfy the BPS condition.

The string and some D3-brane solutions for 1/4 BPS Wilson loops can be found from the supersymmetry conditions [32]. Following the same steps, we study the $1 / 4$ BPS Wilson-'t Hooft loops using D3-branes with both electric and magnetic charges on its world-volume.

\subsection{D3-brane solution}

The Wilson-'t Hooft loops have different representation of the gauge group. In the bulk description, the existence of the D-string of the Wilson-' $t$ Hooft loop makes the general representation difficulty to study. We will consider the case that the $F 1$ strings and $D 1$ stings are in the symmetric representation. The $F 1$ 's and $D 1$ 's form a simple bound state. We will find a D3-brane solutions associated to this Wilson-'t Hooft loops. The Wilson-'t Hooft loop that we consider is localized in the time direction and preserves an $\mathrm{SO}(3) \times \mathrm{SO}(3)$ symmetry. These $\mathrm{SO}(3) \times \mathrm{SO}(3)$ are the isometry of $A d S_{5}$ and $S^{5}$ separately. It is convenient to use the metric (2.2) and fix a static gauge for D3-brane with worldvolume coordinates $(t, \rho, \vartheta, \varphi)$. Then the D3-brane hypersurface in $A d S_{5} \times S^{5}$ is characterized by the equations

$$
\chi=\chi(\rho), \quad \theta=\theta(\rho), \quad \phi=t .
$$

The D3-brane action includes three parts: the Dirac-Born-Infeld action (DBI), WessZumio action (WZ) and boundary term

$$
S=S_{D B I}+S_{W Z}+S_{\text {boudary }}=T_{D_{3}} \int e^{-\Phi} \sqrt{-\operatorname{det}\left(g+2 \pi \alpha^{\prime} F\right)}-T_{D_{3}} \int P\left[C_{4}\right]+S_{\text {boudary }} .
$$

The tension of the D3-brane is

$$
T_{D 3}=\frac{N}{2 \pi^{2} L^{4}} \text {. }
$$

$P\left[C_{4}\right]$ is the pullback of the Ramond-Ramond 4-form potential to the worldvolume of D3brane. With the ansatz (2.13), the DBI action is of the form(absorb a factor of $\frac{2 \pi \alpha^{\prime}}{L^{2}}$ in the definition of $\left.F_{t \rho}, F_{\vartheta \varphi}\right)$

$$
S_{D B I}=\frac{N}{2 \pi^{2}} \int d t d \rho d \vartheta d \varphi \sqrt{\left(\cosh ^{2} \rho-\sin ^{2} \theta\right)\left(1+\sinh ^{2} \rho \chi^{\prime 2}+\theta^{\prime 2}\right)-F_{t \rho}^{2}} \sqrt{\sin ^{4} \chi \sinh ^{4} \rho \sin ^{2} \vartheta+F_{\vartheta \varphi}^{2}} .
$$


Where $F_{t \rho}$ is the elecric field and $F_{\vartheta \varphi}$ is the magnetic field on D3-brane. The ' denotes a derivative with respect to $\rho$. The Wess-Zumino term is given by

$$
S_{W Z}=\frac{2 N}{\pi} \int d t d \rho \sinh ^{4} \rho \sin ^{2} \chi \chi^{\prime} .
$$

It is difficult to solve the equations of motion from this action. We will discuss the supersymmetry equations of the D3-brane from requiring kappa symmetry. These are first-order equations and can be solved easily. These solutions can be checked to satisfy the equations of motion derived from the action. The $\kappa$ symmetry of D-branes is defined as [33-40]

$$
\begin{aligned}
d^{p+1} \xi \Gamma & =-\left.e^{-\Phi} \mathcal{L}_{D B I}^{-1} e^{\mathcal{F}} \wedge X\right|_{\text {vol }}, \\
X & =\bigoplus \Gamma_{(2 n)} K^{n} I,
\end{aligned}
$$

where the operations $I$ and $K$ act on spinors as $I \psi=-i \psi$ and $K \psi=\psi^{*}$, and the notation $\Gamma_{(n)}$ is defined as

$$
\Gamma_{(n)}=\frac{1}{n !} d \xi^{i_{n}} \wedge \ldots \wedge d \xi^{i_{1}} \Gamma_{i_{1} \cdots i_{n}} .
$$

Where $\Gamma_{i_{1} \cdots i_{n}}$ is the pullback of the target space gamma matrice

$$
\Gamma_{i_{1} \cdots i_{n}}=\partial_{i_{1}} X^{m_{1}} \ldots \partial_{i_{n}} X^{m_{n}} \Gamma_{m_{1} \cdots m_{n}} .
$$

The $\kappa$ symmetry of D3-brane with electric and magnetic field on its world-volume is

$$
\Gamma=\mathcal{L}_{D B I}^{-1}\left(\Gamma_{(4)}+L^{2} F_{t \rho} \Gamma_{(2)} K+L^{2} F_{\vartheta \varphi} \tilde{\Gamma}_{(2)} K+L^{4} F_{t \rho} F_{\vartheta \varphi}\right) I .
$$

The projectors $\Gamma_{(4)}, \Gamma_{(2)}$ and $\tilde{\Gamma}_{(2)}$ are

$$
\begin{aligned}
& \Gamma_{(4)}=\tilde{\Gamma}_{(2)} \Gamma_{(2)}=\partial_{t} x^{\mu} \partial_{\rho} x^{\nu} \partial_{\vartheta} x^{\xi} \partial_{\varphi} x^{\varsigma} \gamma_{\mu} \gamma_{\nu} \gamma_{\xi} \gamma_{\zeta}=\left(\gamma_{t}+\gamma_{\phi}\right)\left(\gamma_{\rho}+\chi^{\prime} \gamma_{\chi}+\theta^{\prime} \gamma_{\theta}\right) \gamma_{\vartheta} \gamma_{\varphi} \\
& \Gamma_{(2)}=\partial_{\vartheta} x^{\mu} \partial_{\varphi} x^{\nu} \gamma_{\mu} \gamma_{\nu}=\gamma_{\vartheta} \gamma_{\varphi}, \quad \tilde{\Gamma}_{(2)}=\left(\gamma_{t}+\gamma_{\phi}\right)\left(\gamma_{\rho}+\chi^{\prime} \gamma_{\chi}+\theta^{\prime} \gamma_{\theta}\right) .
\end{aligned}
$$

Using the Vielbeins

$$
\begin{gathered}
e^{0}=L \cosh \rho d t, \quad e^{1}=L d \rho, \quad e^{2}=L \sinh \rho d \chi \\
e^{3}=L \sinh \rho \sin \chi d \vartheta, \quad e^{4}=L \sinh \rho \sin \chi \sin \vartheta d \varphi \\
e^{5}=L d \theta, \quad e^{6}=L \sin \theta d \phi
\end{gathered}
$$

and the ansatz $(2.13)$, the projectors $\Gamma_{(4)}, \Gamma_{(2)}$ and $\tilde{\Gamma}_{(2)}$ are written as

$$
\begin{aligned}
& \Gamma_{(4)}=\tilde{\Gamma}_{(2)} \Gamma_{(2)}=L^{4}\left(\cosh \rho \Gamma_{0}+\sin \theta \Gamma_{6}\right)\left(\Gamma_{1}+\sinh \rho \chi^{\prime} \Gamma_{2}+\theta^{\prime} \Gamma_{5}\right) \sinh ^{2} \rho \sin ^{2} \chi \sin \vartheta \Gamma_{34}, \\
& \Gamma_{(2)}=L^{2} \sinh ^{2} \rho \sin ^{2} \chi \sin \vartheta \Gamma_{34}, \quad \tilde{\Gamma}_{(2)}=L^{2}\left(\cosh \rho \Gamma_{0}+\sin \theta \Gamma_{6}\right)\left(\Gamma_{1}+\sinh \rho \chi^{\prime} \Gamma_{2}+\theta^{\prime} \Gamma_{5}\right) .
\end{aligned}
$$

The Killing spinor for the metric $(2.2)$ is

$$
\epsilon=e^{-\frac{i}{2} \rho \Gamma_{*} \Gamma_{1}} e^{-\frac{i}{2} t \Gamma_{*} \Gamma_{0}} e^{\frac{1}{2} \chi \Gamma_{12}} e^{\frac{1}{2} \vartheta F_{23}} e^{\frac{1}{2} \varphi \Gamma_{34}} e^{-\frac{i}{2} \theta \Gamma_{*} \Gamma_{5}} e^{\frac{1}{2} \phi F_{56}} \epsilon_{0} .
$$

Similar to the Wilson loop case, we make the following ansatz on $\epsilon_{0}$ for $1 / 4$ BPS Wilson-'t Hooft loops:

$$
K \epsilon_{0}=-e^{i \alpha} \Gamma_{01} \epsilon_{0}, \quad \Gamma_{6} \epsilon_{0}=-i \Gamma_{12345} \epsilon_{0}
$$


These conditions are different with the conditions (2.10) of Wilson loop with extra factor $e^{i \alpha}$. The $\alpha$ is the real number which is similar to the angle. When $\alpha=0$, this conditions reduce to the 1/4 BPS Wilson loop conditions (2.10) corresponding the pure electric case.

Putting the $\phi=t$ in the expression (2.30), the Killing spinors can be rewritten as

$$
\epsilon=e^{-\frac{i}{2} \rho \Gamma_{*} \Gamma_{1}} e^{-\frac{i}{2} \theta \Gamma_{*} \Gamma_{5}} e^{\frac{1}{2} \chi \Gamma_{12}} e^{\frac{1}{2} \vartheta F_{23}} e^{\frac{1}{2} \varphi \Gamma_{34}} \epsilon_{0}=e^{-\frac{i}{2} \rho \Gamma_{*} \Gamma_{1}} e^{-\frac{i}{2} \theta \Gamma_{*} \Gamma_{5}} e^{\frac{1}{2} \chi \Gamma_{12}} M \epsilon_{0} .
$$

Where

$$
M=e^{\frac{1}{2} \vartheta F_{23}} e^{\frac{1}{2} \varphi \Gamma_{34}} .
$$

We note that the $\epsilon_{0}$ and $M \epsilon_{0}$ satisfy the same constraints.

The differential equations for the 1/4 BPS Wilson-'t Hooft loops come from the projection relation

$$
\Gamma \epsilon=\epsilon .
$$

After moving the matrix $e^{-\frac{i}{2} \rho \Gamma_{*} \Gamma_{1}} e^{-\frac{i}{2} \theta \Gamma_{*} \Gamma_{5}} e^{\frac{1}{2} \chi \Gamma_{12}}$ to the left of the projector and applying the constrains (2.31), we obtain the 8 differential equations for the $\theta, \chi, F_{t \rho}$ and $F_{\vartheta \varphi}$

$$
\begin{aligned}
& \Gamma_{0345}: 0=\sinh ^{2} \rho \sin ^{2} \chi \sin \vartheta\left(e^{i \alpha} F_{t \rho} \sinh \rho \cos \chi \sin \theta-\theta^{\prime}\left(\cosh ^{2} \rho-\sin ^{2} \theta\right)\right)-i e^{i \alpha} F_{\vartheta \varphi} \sinh ^{3} \rho \cos \chi \sin \theta \chi^{\prime} \\
& \Gamma_{*} \Gamma_{5}: 0=\sinh ^{2} \rho \sin ^{2} \chi \sin \vartheta\left(e^{i \alpha} F_{t \rho} \sinh \rho \sin \chi \sin \theta-\chi^{\prime} \sinh ^{2} \rho \sin \theta \cos \theta\right)-i e^{i \alpha} F_{\vartheta \varphi} \sinh ^{3} \rho \sin \chi \sin \theta \chi^{\prime} \\
& \Gamma_{0234}: 0=\left(\cosh ^{2} \rho-\sin ^{2} \theta\right) \sin \chi+\chi^{\prime} \cosh \rho \sinh \rho \cos \chi \cos ^{2} \theta \\
& \Gamma_{12} \quad: 0=\sinh ^{2} \rho \sin ^{2} \chi \sin \vartheta\left(e^{i \alpha} F_{t \rho} \sinh \rho \cos \chi \cos \theta+\theta^{\prime} \sin \theta \cos \theta+\cosh \rho \sinh \rho\right) \\
& \quad-i e^{i \alpha} F_{\vartheta \varphi} \sinh ^{3} \rho \cos \chi \cos \theta \chi^{\prime} \\
& \Gamma_{15} \quad: 0=\chi^{\prime} \cosh \rho \sinh \rho \cos \chi \sin \theta \cos \theta-\theta^{\prime} \cosh \rho \sinh \rho \sin \chi+\sin \chi \sin \theta \cos \theta \\
& \Gamma_{25} \quad: 0=\sinh ^{2} \rho \sin ^{2} \chi \sin \vartheta\left(e^{i \alpha} F_{t \rho} \cosh \rho \sin \theta+\cos \chi \sin \theta \cos \theta-\chi^{\prime} \cosh \rho \sinh \rho \sin \chi \sin \theta \cos \theta-\right. \\
&\left.\quad \theta^{\prime} \cosh \rho \sinh ^{2} \rho \cos \chi\right)-i e^{i \alpha} F_{\vartheta \varphi} \sinh ^{2} \rho \cosh \rho \sin \theta \chi^{\prime} \\
& \Gamma_{0134} \quad: 0=\sinh ^{2} \rho \sin ^{2} \chi \sin \vartheta\left(e^{i \alpha} F_{t \rho} \cosh \rho \cos \theta+\left(\cosh { }^{2} \rho-\sin ^{2} \theta\right) \cos \chi-\chi^{\prime} \cosh \rho \sinh \rho \sin \chi \cos ^{2} \theta\right) \\
& \quad-i e^{i \alpha} F_{\vartheta \varphi} \sinh ^{2} \rho \cosh \rho \cos \theta \chi^{\prime} \\
& \quad:-\left(\frac{1}{L^{4}}\right) \mathcal{L}_{D B I}=\sinh { }^{2} \rho \sin ^{2} \chi \sin \vartheta\left(e^{i \alpha} F_{t \rho} \sinh \rho \sin \chi \cos \theta+\chi^{\prime} \sinh ^{2} \rho \sin ^{2} \theta\right) \\
& \quad-i e^{i \alpha} F_{\vartheta \varphi} \sinh ^{3} \rho \sin \chi \cos \theta \chi^{\prime}-i e^{i \alpha} F_{\vartheta \varphi} \frac{\cos \theta \sinh \rho}{\sin \chi} \chi^{\prime}+i F_{t \rho} F_{\vartheta \varphi}
\end{aligned}
$$

These 8 differential equations have 3 independent differential equations

$$
\begin{aligned}
\theta^{\prime} & =-\tan \theta \tanh \rho, \\
\chi^{\prime} \cot \chi & =-\frac{\cosh ^{2} \rho-\sin ^{2} \theta}{\cosh \rho \sinh \rho \cos ^{2} \theta}, \\
e^{i \alpha} F_{t \rho}-i e^{i \alpha} \chi^{\prime} \sin ^{2} \rho \tilde{F}_{\vartheta \varphi} & =-\frac{\cosh ^{2} \rho-\sin ^{2} \theta}{\cosh \rho \cos \theta \cos \chi},
\end{aligned}
$$

where

$$
\tilde{F}_{\vartheta \varphi}=\frac{F_{\vartheta \varphi}}{\sinh ^{2} \rho \sin ^{2} \chi \sin \vartheta} .
$$

We solve for $\chi, \theta, F_{t \rho}$ and $\tilde{F}_{\vartheta \varphi}$ from these equations. The solutions to equations (2.36) and (2.37) are

$$
\sin \theta=\frac{C_{1}}{\cosh \rho}, \quad \sin \chi=C_{2} \frac{\operatorname{coth} \rho}{\sqrt{\cosh ^{2} \rho-C_{1}^{2}}} .
$$


Where the $C_{1}$ and $C_{2}$ are two constants. The $C_{1}$ is related to the angular momentum carried by the D3-brane. The $C_{2}$ is related to the electric and magnetic charge of D3brane. The electric and magnetic field $F_{t \rho}, \tilde{F}_{\vartheta \varphi}$ can be solved from the equation (2.38). We can obtain two equations from this complex equation (2.38) by making the real part and imaginary part equal separately. Finally, we get the results

$$
F_{t \rho}=-\cos \alpha\left(\frac{\cosh ^{2} \rho-\sin ^{2} \theta}{\cosh \rho \cos \theta \cos \chi}\right), \quad \tilde{F}_{\vartheta \varphi}=\frac{\cos \theta}{\sinh \rho \sin \chi} \sin \alpha .
$$

When $\cos \alpha=0$, the $1 / 4$ BPS Wilson-'t Hooft loops become the magnetic 't Hooft loops.

\subsection{Conserved charges}

The are two constants $C_{1}, C_{2}$ in the solutions. The $C_{1}$ is related to the angular momentum $\mathrm{J}$ around the $S^{2}$ in the $S^{5}$. The $C_{2}$ is related to the electric and magnetic charge carried by the D3-brane.

The electric charge $\mathrm{k}$ is the conjugate momentum to the electric field

$$
k=\Pi=\frac{2 \pi \alpha^{\prime}}{L^{2}} T_{D 3} \int d \vartheta d \varphi \frac{\delta \mathcal{L}}{\delta F_{t \rho}}=\frac{4 N}{\sqrt{\lambda}} C_{2} \cos \alpha .
$$

The integer charge $k$ corresponds to the number of coincident F1 stings. We also have the magnetic charge $m$

$$
m=\frac{1}{2 \pi} \frac{L^{2}}{2 \pi \alpha^{\prime}} \int d \vartheta d \varphi F_{\vartheta \varphi}=\frac{\sqrt{\lambda}}{\pi} C_{2} \sin \alpha .
$$

The integer magnetic charge $m$ is the number of D1-branes immersed in the D3-branes. From equations (2.42) and (2.43), we obtain the $\mathrm{k}$ and $m$ satisfy the following relation

$$
\frac{k^{2} \lambda}{16 N^{2}}+\frac{(m \pi)^{2}}{\lambda}=C_{2}^{2}
$$

Using the dual coupling constant $\tilde{\lambda}=\frac{16 \pi^{2} N^{2}}{\lambda}$, the $C_{2}$ can be rewritten as

$$
C_{2}^{2}=\frac{k^{2} \lambda}{(4 N)^{2}}+\frac{m^{2} \tilde{\lambda}}{(4 N)^{2}}
$$

We then discuss the energy and angular momentum relation. The angular momentum $\mathrm{J}$ is

$$
\begin{aligned}
J & =2 T_{D 3} \int d \vartheta d \varphi d \rho \frac{\delta \mathcal{L}}{\delta \dot{\phi}} \\
& =-\frac{4 N}{\pi} \int d \rho \frac{\sinh ^{2} \rho \sin ^{2} \chi \sin ^{2} \theta\left(1+\sinh ^{2} \rho \chi^{\prime 2}+\theta^{\prime 2}\right) \sqrt{1+\tilde{F}_{\vartheta \varphi}^{2}}}{\sqrt{\left(\cosh ^{2} \rho-\sin ^{2} \theta\right)\left(1+\sinh ^{2} \rho \chi^{\prime 2}+\theta^{\prime 2}\right)-F_{t \rho}^{2}}} .
\end{aligned}
$$

The energy contribution from DBI action and the Wess-Zumino term is

$$
\begin{aligned}
E_{D . W} & =2 T_{D 3} \int d \vartheta d \varphi d \rho \frac{\delta\left(\mathcal{L}_{D B I}+\mathcal{L}_{W Z}\right)}{\delta \dot{t}} \\
& =\frac{4 N}{\pi} \int d \rho \frac{\sinh ^{2} \rho \sin ^{2} \chi \cosh ^{2} \rho\left(1+\sinh ^{2} \rho \chi^{\prime 2}+\theta^{\prime 2}\right) \sqrt{1+\tilde{F}_{\vartheta \varphi}^{2}}}{\sqrt{\left(\cosh ^{2} \rho-\sin ^{2} \theta\right)\left(1+\sinh ^{2} \rho \chi^{\prime 2}+\theta^{\prime 2}\right)-F_{t \rho}^{2}}}+\frac{4 N}{\pi} \int d \rho \sinh ^{4} \rho \sin ^{2} \chi \chi^{\prime}
\end{aligned}
$$


The other energy term comes from the the electric and magnetic field. That is

$$
\begin{aligned}
E_{L . T .}= & \frac{4 N}{\pi} \int d \rho\left(-\frac{\delta \mathcal{L}_{D B I}}{F_{\vartheta \varphi}} F_{\vartheta \varphi}+\frac{\delta \mathcal{L}_{D B I}}{F_{t \rho}} F_{t \rho}\right) \\
= & -\frac{4 N}{\pi} \int d \rho \frac{\sinh ^{2} \rho \sin ^{2} \chi \tilde{F}_{\vartheta \varphi}^{2} \sqrt{\left(\cosh ^{2} \rho-\sin ^{2} \theta\right)\left(1+\sinh ^{2} \rho \chi^{\prime 2}+\theta^{\prime 2}\right)-F_{t \rho}^{2}}}{\sqrt{1+\tilde{F}_{\vartheta \varphi}^{2}}} \\
& -\frac{4 N}{\pi} \int d \rho \frac{\sinh ^{2} \rho \sin ^{2} \chi F_{t \rho}^{2} \sqrt{1+\tilde{F}_{\vartheta \varphi}^{2}}}{\sqrt{\left(\cosh ^{2} \rho-\sin ^{2} \theta\right)\left(1+\sinh ^{2} \rho \chi^{\prime 2}+\theta^{\prime 2}\right)-F_{t \rho}^{2}}}
\end{aligned}
$$

Using the above expressions, it is easy to find $J+E_{D . W}+E_{L . T .}=0$. This is consistent with the BPS condition.

\section{$3 \quad 1 / 4$ BPS circular Wilson-'t Hooft loop}

\subsection{Review of the 1/4 BPS circular Wilson loop}

In this section, we will discuss $1 / 4$ BPS circular Wilson-'t Hooft loop. We first give a briefly review of the BPS circular Wilson loop [41-43]. The 1/4 BPS circular Wilson loop can be parameterized by

$$
x^{1}=R \cos \alpha, \quad x^{2}=R \sin \alpha
$$

on the boundary of $A d S_{5}$. It couples to a linear combination scalar

$$
\Phi(\alpha)=\Phi_{3} \cos \theta_{0}+\sin \theta_{0}\left(\Phi_{1} \cos \alpha+\Phi_{2} \sin \alpha\right)
$$

with an arbitrary fixed $\theta_{0}$. The loop may be written as (in Euclidean signature)

$$
W_{\theta_{0}}=\operatorname{Tr} \mathcal{P} \exp \left[\oint\left(i A_{\mu}(\alpha) \dot{x}^{\mu}+|\dot{x}| \Phi(\alpha)\right) d \alpha\right] .
$$

The Wilson loop will be given by the sum of all non-interacting diagrams which is written in terms of a 0-dimensional Hermitian Gaussian matrix model. The expectation value of this Wilson loop is given by the matrix model as

$$
\left\langle W_{\theta_{0}}\right\rangle=\frac{1}{Z} \int \mathcal{D} M \frac{1}{N} \operatorname{Tr} e^{M} e^{-\frac{2 N}{\lambda^{\prime}} \operatorname{Tr} M^{2}}=\frac{1}{N} L_{N-1}^{1}\left(-\frac{\lambda^{\prime}}{4 N}\right) \exp \left[\frac{\lambda^{\prime}}{8 N}\right] .
$$

Where $L_{N-1}^{1}$ is a Laguerre polynomial and $\lambda^{\prime}=\lambda \cos ^{2} \theta_{0}$. In planar limit, the expectation value is given by

$$
\left\langle W_{\theta_{0}, \text { planar }}\right\rangle=\frac{2}{\sqrt{\lambda^{\prime}}} I_{1}\left(\sqrt{\lambda^{\prime}}\right) .
$$

Where $I_{1}$ is a modified Bessel function.

To discuss the relevant string solutions in the dual $A d S_{5} \times S^{5}$ space, it is useful to adopt the following metric on $A d S_{5} \times S^{2}$ (the other directions on $S^{5}$ are omitted)

$$
\frac{d s^{2}}{L^{2}}=-d \chi^{2}+\cos ^{2} \chi\left(d \rho^{2}+\sinh ^{2} \rho d \psi^{2}\right)+\sin ^{2} \chi\left(d \sigma^{2}+\sinh ^{2} \sigma d \varphi^{2}\right)+d \theta^{2}+\sin ^{2} \theta d \phi^{2} .
$$


The metric has Lorentzian signature. The Lorentzian can be used for the supersymmetry analysis.

The string describing the Wilson loop (3.3) will be at $\chi=0$ and end at $\rho \rightarrow \infty$. Using the ansatz

$$
\rho=\rho(\sigma), \quad \psi(\tau)=\tau, \quad \theta=\theta(\tau), \quad \phi(\tau)=\tau, \quad \chi=0,
$$

the string action is

$$
\mathcal{S}=\frac{L^{2}}{4 \pi \alpha^{\prime}} \int d \sigma d \tau\left[\rho^{\prime 2}+\sinh ^{2} \rho+\theta^{\prime 2}+\sin ^{2} \theta\right]
$$

The equations of motion are

$$
\begin{aligned}
\rho^{\prime \prime} & =\sinh \rho \cosh \rho, \\
\theta^{\prime \prime} & =\sin \theta \cos \theta .
\end{aligned}
$$

Two solutions with these boundary conditions were found [43]:

$$
\phi=\psi, \quad \sinh \rho(\sigma)=\frac{1}{\sinh \sigma}, \quad \sin \theta=\frac{1}{\cosh \left(\sigma_{0} \pm \sigma\right)} .
$$

Here $\sigma$ is a world-sheet coordinate. The constant $\sigma_{0}$ is fixed by the boundary condition that at $\sigma=0$

$$
\cos \theta_{0}=\tanh \sigma_{0}
$$

From these solutions, the classical action is

$$
\mathcal{S}=\mp \cos \theta_{0} \sqrt{\lambda} .
$$

The two signs correspond to a string extended over the north or south pole of $S^{2}$. The solutions preserve $1 / 4$ of the supersymmetries. This can be seen from supersymmetry analysis. The Killing spinor on the relevant component of the metric (3.6) is

$$
\epsilon=e^{-\frac{i}{2} \rho \Gamma_{\star} \Gamma_{1}} e^{\frac{1}{2} \psi \Gamma_{12}} e^{-\frac{i}{2} \theta \Gamma_{\star} \Gamma_{5}} e^{\frac{1}{2} \phi \Gamma_{56}} \epsilon_{0} .
$$

Then it can obtain two compatible constraints.

$$
\left(\Gamma_{12}+\Gamma_{56}\right) \epsilon_{0}=0
$$

and

$$
K \epsilon_{0}=-\left(\cos \theta_{0} \Gamma_{12}+\sin \theta_{0} \Gamma_{16}\right) \epsilon_{0} .
$$

The construction of the $1 / 4$ BPS D3-brane describing the circular Wilson loop in the $\mathrm{k}$-th symmetric representation can be found in [32]. For k-th symmetric representation, the expectation value is

$$
\left\langle W_{k^{\prime}}\right\rangle=\exp \left[2 N\left(k^{\prime} \sqrt{1+k^{\prime 2}}+\operatorname{arcsinh} k^{\prime}\right)\right]
$$

with

$$
k^{\prime}=\frac{k \cos \theta_{0} \sqrt{\lambda}}{4 N} .
$$

The $\theta_{0}=0$ corresponds to the $1 / 2$ BPS Wilson loop (1.8).

We will turn on the magnetic flux on the D3-brane worldvolume to find a $1 / 4$ BPS circular Wilson-'t Hooft loop with D3-brane description in the symmetric representation. 


\subsection{D3-brane solution}

We use the Lorentzian signature metric (3.6) to obtain the equations of motion from the supersymmetry analysis. In this Lorentzian signature metric, the brane has extra factor of $i$ in the projector equation and electric field. We parameterize the D3 brane worldvolume by $\{\rho, \psi, \sigma, \varphi\}$. The ansatz for the $1 / 4$ BPS circular Wilson-'t Hooft loop is to take $\chi=\chi(\rho), \theta=\theta(\rho), \psi=\phi$. The killing spinor with Lorentzian signature metric (3.6) can be written as

$$
\epsilon=e^{-\frac{i}{2} \chi \Gamma_{\star} \Gamma_{0}} e^{-\frac{i}{2} \rho \Gamma_{\star} \Gamma_{1}} e^{\frac{1}{2} \psi \Gamma_{12}} e^{-\frac{1}{2} \sigma \Gamma_{03}} e^{\frac{1}{2} \varphi \Gamma_{34}} e^{-\frac{i}{2} \theta \Gamma_{\star} \Gamma_{5}} e^{\frac{1}{2} \phi \Gamma_{56}} \epsilon_{0} .
$$

The DBI action is of the form

$$
\mathcal{L}_{D B I}=L^{4} \sin ^{2} \chi \sinh \sigma \sqrt{\left(\left(-\chi^{\prime 2}+\theta^{\prime 2}+\cos ^{2} \chi\right)\left(\cos ^{2} \chi \sinh ^{2} \rho+\sin ^{2} \theta\right)+F_{\rho \psi}^{2}\right)\left(1-\tilde{F}_{\sigma \varphi}^{2}\right)},
$$

where we define

$$
\tilde{F}_{\sigma \varphi}=\frac{F_{\sigma \varphi}}{\sin ^{2} \chi \sinh \sigma} .
$$

From the general $\kappa$ symmetry expression (2.18), we obtain

$$
\Gamma=\mathcal{L}_{D B I}^{-1}\left(i \Gamma_{(4)}-L^{2} F_{\rho \psi} \Gamma_{(2)} K+i L^{2} F_{\sigma \varphi} \tilde{\Gamma}_{(2)} K-L^{4} F_{\rho \psi} F_{\sigma \varphi}\right) I .
$$

The vielbeins for the metric are

$$
\begin{gathered}
e^{0}=L d \chi, \quad e^{1}=L \cos \chi d \rho, \quad e^{2}=L \cos \chi \sinh \rho d \psi, \\
e^{3}=L \sin \chi d \sigma, \quad e^{4}=L \sin \chi \sinh \sigma d \varphi \\
e^{5}=L d \theta, \quad e^{6}=L \sin \theta d \phi .
\end{gathered}
$$

Then the projectors $\Gamma_{(4)}, \tilde{\Gamma}_{(2)}$ and $\Gamma_{(2)}$ can be written as

$$
\begin{aligned}
\Gamma_{(4)} & =\left(\gamma_{\rho}+\chi^{\prime} \gamma_{\chi}+\theta^{\prime} \gamma_{\theta}\right)\left(\gamma_{\psi}+\gamma_{\phi}\right) \gamma_{\sigma} \gamma_{\varphi} \\
& =L^{4}\left(\cos \chi \Gamma_{1}+\chi^{\prime} \Gamma_{0}+\theta^{\prime} \Gamma_{5}\right)\left(\cos \chi \sinh \rho \Gamma_{2}+\sin \theta \Gamma_{6}\right) \sin ^{2} \chi \sinh \sigma \Gamma_{34}, \\
\tilde{\Gamma}_{(2)} & =\left(\gamma_{\rho}+\chi^{\prime} \gamma_{\chi}+\theta^{\prime} \gamma_{\theta}\right)\left(\gamma_{\psi}+\gamma_{\phi}\right)=L^{2}\left(\cos \chi \Gamma_{1}+\chi^{\prime} \Gamma_{0}+\theta^{\prime} \Gamma_{5}\right)\left(\cos \chi \sinh \rho \Gamma_{2}+\sin \theta \Gamma_{6}\right), \\
\Gamma_{(2)} & =\gamma_{\sigma} \gamma_{\varphi}=L^{2} \sin ^{2} \chi \sinh \sigma \Gamma_{34} .
\end{aligned}
$$

The projector $\Gamma$ does not depend on $\psi$. We can eliminate the dependence on $\psi$ by imposing

$$
\left(\Gamma_{12}+\Gamma_{56}\right) \epsilon_{0}=0 .
$$

After imposing this constraint, the Killing spinor (3.19) can be reduced to

$$
\epsilon=e^{-\frac{i}{2} \chi \Gamma_{\star} \Gamma_{0}} e^{-\frac{i}{2} \rho \Gamma_{\star} \Gamma_{1}} e^{-\frac{1}{2} \sigma \Gamma_{03}} e^{\frac{1}{2} \varphi \Gamma_{34}} e^{-\frac{i}{2} \theta \Gamma_{\star} \Gamma_{5}} \epsilon_{0}=e^{-\frac{i}{2} \chi \Gamma_{\star} \Gamma_{0}} e^{-\frac{i}{2} \rho \Gamma_{\star} \Gamma_{1}} e^{-\frac{i}{2} \theta \Gamma_{\star} \Gamma_{5}} M \epsilon_{0} .
$$

Where $M$ is defined as

$$
M=e^{-\frac{1}{2} \sigma \Gamma_{03}} e^{\frac{1}{2} \varphi \Gamma_{34}} .
$$

To obtain the 1/4 BPS circular Wilson-'t Hooft loop, we impose another condition

$$
K \epsilon_{0}=-e^{i \gamma}\left(\cos \theta_{0} \Gamma_{12}+\sin \theta_{0} \Gamma_{16}\right) \epsilon_{0} .
$$


This condition is different with the condition (3.16) of Wilson loop with extra factor $e^{i \gamma}$. The $\gamma$ is the real number and connected with the electric and magnetic charge carried by the D3-brane. The differential equations for the 1/4 BPS Wilson-'t Hooft loops come from the projection relation

$$
\Gamma \epsilon=\epsilon
$$

Moving the matrix $e^{-\frac{i}{2} \chi \Gamma_{\star} \Gamma_{0}} e^{-\frac{i}{2} \rho \Gamma_{\star} \Gamma_{1}} e^{-\frac{i}{2} \theta \Gamma_{\star} \Gamma_{5}}$ to the left of $\Gamma$, we obtain the 8 first order differential equations for $\theta, \chi, F_{\rho \psi}$ and $\tilde{F}_{\sigma \varphi}$

$$
\begin{aligned}
& \Gamma_{0234}: 0=i e^{i \gamma} F_{\rho \psi} \sin \chi \sin \theta \sin \theta_{0}-i e^{i \gamma} \tilde{F}_{\sigma \varphi}\left(\sinh \rho \sin \chi \cos \chi-\cosh \rho \chi^{\prime}\right) \sin \chi \sin \theta \sin \theta_{0} \\
& +\chi^{\prime} \sinh \rho\left(\cos ^{2} \chi-\sin ^{2} \theta\right)+\cosh \rho \sin \chi \cos \chi \sin ^{2} \theta \\
& \Gamma_{\star} \Gamma_{5} \quad: 0=i e^{i \gamma} F_{\rho \psi} \sin \chi \sin \theta \cos \theta_{0}-i e^{i \gamma} \tilde{F}_{\sigma \varphi}\left(\sinh \rho \sin \chi \cos \chi-\cosh \rho \chi^{\prime}\right) \sin \chi \sin \theta \cos \theta_{0} \\
& -\chi^{\prime} \cosh \rho \sin \theta \cos \theta+\sinh \rho \sin \chi \cos \chi \sin \theta \cos \theta \\
& \Gamma_{1234}: 0=i e^{i \gamma} F_{\rho \psi} \sinh \rho \cos \chi \sin \theta \sin \theta_{0}-i e^{i \gamma} \tilde{F}_{\sigma \varphi}\left(\sinh \rho \sin \chi \cos \chi-\cosh \rho \chi^{\prime}\right) \sinh \rho \cos \chi \sin \theta \sin \theta_{0} \\
& +i e^{i \gamma} F_{\rho \psi} \cosh \rho \cos \chi \cos \theta \cos \theta_{0}-i e^{i \gamma} \tilde{F}_{\sigma \varphi}\left(\sinh \rho \sin \chi \cos \chi-\cosh \rho \chi^{\prime}\right) \cosh \rho \cos \chi \cos \theta \cos \theta_{0} \\
& -\chi^{\prime} \sinh ^{2} \rho \sin \chi \cos \chi-\theta^{\prime} \sin \theta \cos \theta+\sinh \rho \cosh \rho \cos ^{2} \chi \\
& \Gamma_{2345} \quad 0=i e^{i \gamma} F_{\rho \psi} \sinh \rho \cos \chi \sin \theta \cos \theta_{0}-i e^{i \gamma} \tilde{F}_{\sigma \varphi}\left(\sinh \rho \sin \chi \cos \chi-\cosh \rho \chi^{\prime}\right) \sinh \rho \cos \chi \sin \theta \cos \theta_{0} \\
& -i e^{i \gamma} F_{\rho \psi} \cosh \rho \cos \chi \cos \theta \sin \theta_{0}+i e^{i \gamma} \tilde{F}_{\sigma \varphi}\left(\sinh \rho \sin \chi \cos \chi-\cosh \rho \chi^{\prime}\right) \cosh \rho \cos \chi \cos \theta \sin \theta_{0} \\
& -\theta^{\prime} \sinh \rho \cosh \rho \cos ^{2} \chi-\cos ^{2} \chi \sin \theta \cos \theta \\
& -\chi^{\prime} \sinh \rho \cosh \rho \sin \chi \cos \chi+\cos ^{2} \chi\left(\sinh ^{2} \rho+\sin ^{2} \theta\right) \\
& \Gamma_{05} \quad: 0=i e^{i \gamma} F_{\rho \psi} \sinh \rho \cos \chi \cos \theta \sin \theta_{0}-i e^{i \gamma} \tilde{F}_{\sigma \varphi}\left(\sinh \rho \sin \chi \cos \chi-\cosh \rho \chi^{\prime}\right) \sinh \rho \cos \chi \cos \theta \sin \theta_{0} \\
& -i e^{i \gamma} F_{\rho \psi} \cosh \rho \cos \chi \sin \theta \cos \theta_{0}+i e^{i \gamma} \tilde{F}_{\sigma \varphi}\left(\sinh \rho \sin \chi \cos \chi-\cosh \rho \chi^{\prime}\right) \cosh \rho \cos \chi \sin \theta \cos \theta_{0} \\
& +\theta^{\prime}\left(\sinh ^{2} \rho \cos ^{2} \chi+\sin ^{2} \theta\right) \\
& \Gamma_{15} \quad: 0=i e^{i \gamma} F_{\rho \psi} \sin \chi \cos \theta \sin \theta_{0}-i e^{i \gamma} \tilde{F}_{\sigma \varphi}\left(\sinh \rho \sin \chi \cos \chi-\cosh \rho \chi^{\prime}\right) \sin \chi \cos \theta \sin \theta_{0} \\
& -\chi^{\prime} \sinh \rho \sin \theta \cos \theta+\theta^{\prime} \sinh \rho \sin \chi \cos \chi+\cosh \rho \sin \chi \cos \chi \sin \theta \cos \theta \\
& 1: 1=-i L^{4} \mathcal{L}_{D B I}^{-1} \sin ^{2} \chi \sinh \sigma\left(i e^{i \gamma} F_{\rho \psi} \sin \chi \cos \theta \cos \theta_{0}-i e^{i \gamma} \tilde{F}_{\sigma \varphi}\left(\sin \chi \cos \theta \cos \theta_{0}\right.\right. \\
& \left.-\frac{\cos \theta}{\sin \chi \cos \theta_{0}}\right)\left(\sinh \rho \sin \chi \cos \chi-\cosh \rho \chi^{\prime}\right)+\chi^{\prime} \cosh \rho \sin ^{2} \theta-\sinh \rho \sin \chi \cos \chi \sin ^{2} \theta \\
& \left.-F_{\rho \psi} \tilde{F}_{\sigma \varphi}\right) \text {. }
\end{aligned}
$$

These equations are consistent with each other. We obtain three independent equations

$$
\begin{gathered}
\theta^{\prime}=A \cos ^{2} \chi \cos \theta \quad, \quad \chi^{\prime}=A \sin \chi \cos \chi \sin \theta \\
e^{i \gamma} F_{\rho \psi}-e^{i \gamma} \tilde{F}_{\sigma \varphi}\left(\sinh \rho \sin \chi \cos \chi-\cosh \rho \chi^{\prime}\right)=-i \frac{\cos \chi \cos \theta}{\cos \theta_{0}}(A \cosh \rho \sin \theta-\sinh \rho) .
\end{gathered}
$$

Where

$$
A=\frac{\sinh \rho \cos \theta \sin \theta_{0}-\cosh \rho \sin \theta \cos \theta_{0}}{\left(\cos ^{2} \chi-\sin ^{2} \theta\right) \sinh \rho \cos \theta_{0}+\cosh \rho \sin \theta \cos \theta \sin \theta_{0}} .
$$

The electric field $F_{\rho \psi}$ and magnetic field $\tilde{F}_{\sigma \varphi}$ can be solved from the equation (3.34) by taking the real part and imaginary part equal separately. Then we then get the result

$$
F_{\rho \psi}=-i \frac{\cos \chi \cos \theta}{\cos \theta_{0}}(A \cosh \rho \sin \theta-\sinh \rho) \cos \gamma, \quad \tilde{F}_{\sigma \varphi}=-\frac{\cos \theta}{\sin \chi \cos \theta_{0}} \sin \gamma
$$


We can obtain the $1 / 4$ BPS Wilson loop solution by setting $\gamma=0$. When $\cos \gamma=0$, these solutions are reduced to the 't Hooft loop.

From the equations (3.33), we can get the following results

$$
\begin{aligned}
\sin \chi \cos \theta & =C, \\
\cos \chi\left(\cosh \rho \cos \theta \sin \theta_{0}-\sinh \rho \sin \theta \cos \theta_{0}\right) & =D .
\end{aligned}
$$

The solution not to be singular at the point $\rho=0$ require the $\mathrm{D}$ satisfying the relation

$$
D= \pm \sin \theta_{0} \sqrt{1-C^{2}}
$$

where the,+- signs correspond to taking $\theta=0$ or $\theta=\pi$ at $\rho=0$ respectively. These solutions in Lorentzian space are unphysical. From these solutions we know that the expectation value of the $1 / 4$ BPS Wilson-'t Hooft loop for the fixed $\theta_{0}$ depends on the $C, \gamma$ value.

\subsection{The 1/4 BPS Wilson-'t Hooft loop expectation value}

We now discuss the expectation value of $1 / 4$ BPS Wilson-'t Hooft loop $\left\langle W_{\theta_{0}}^{W H}(C, \gamma)\right\rangle$. We don't know how to calculate it from Super-Yang-Mills theory since we have to work in a background with magnetic monopole. The $\mathrm{SL}(2, Z)$ duality permits us to get the answer. Thanks to the AdS/CFT duality, we can calculate the expectation value of $1 / 4$ BPS Wilson't Hooft loop using the D3-brane action. We first point out that the expectation value is independent of the $\gamma$ value. Then we use the $1 / 4$ BPS Wilson loop result to obtain the expectation value of $1 / 4$ BPS Wilson-'t Hooft loop.

\subsubsection{The expression of expectation value}

The total on-shell D3-brane action of 1/4 BPS Wilson-'t Hooft loop $S_{\theta_{0}}^{W H}(C, \gamma)$ includes four terms: the DBI action $S_{D B I}$, the Wess-Zumino action $S_{W Z}$, the boundary term $S_{F}$ comes from the Legendre transform of the gauge field and the other boundary term $S_{\theta}$ comes from the Legendre transform of the scalar field $\theta^{\prime}$

$$
S_{\theta_{0}}^{W H}(C, \gamma)=S_{D B I}+S_{W Z}+S_{F}+S_{\theta},
$$

where the DBI action is

$$
\begin{aligned}
S_{D B I} & =T_{D 3} \int d \rho d \psi d \sigma d \varphi \mathcal{L}_{D B I} \\
& =\frac{N}{2 \pi^{2}} \int d \rho d \psi d \sigma d \varphi \sin ^{2} \chi \sinh \sigma \sqrt{\left(\left(-\chi^{\prime 2}+\theta^{\prime 2}+\cos ^{2} \chi\right)\left(\cos ^{2} \chi \sinh ^{2} \rho+\sin ^{2} \theta\right)+F_{\rho \psi}^{2}\right)\left(1-\tilde{F}_{\sigma \varphi}^{2}\right)} \\
& =\frac{-i N}{2 \pi^{2}} \int d \rho d \psi d \sigma d \varphi \sin ^{3} \chi \sinh \sigma \cos \chi(A \cosh \rho \sin \theta-\sinh \rho)\left(1-\left(\frac{\cos \theta}{\sin \chi \cos \theta_{0}}\right)^{2} \sin ^{2} \gamma\right)(3.41)
\end{aligned}
$$

and the Legendre transform of the gauge field is

$$
\begin{aligned}
S_{F} & =-T_{D 3} \int d \rho d \psi d \sigma d \varphi \frac{\delta \mathcal{L}_{D B I}}{\delta F_{\rho \psi}} F_{\rho \psi} \\
& =\frac{-i N}{2 \pi^{2}} \int d \rho d \psi d \sigma d \varphi \sin ^{3} \chi \sinh \sigma \cos \chi(A \cosh \rho \sin \theta-\sinh \rho)\left(-\left(\frac{\cos \theta}{\sin \chi \cos \theta_{0}}\right)^{2} \cos ^{2} \gamma\right)
\end{aligned}
$$


Then

$$
S_{D B I}+S_{F}=\frac{-i N}{2 \pi^{2}} \int d \rho d \psi d \sigma d \varphi \sin ^{3} \chi \sinh \sigma \cos \chi(A \cosh \rho \sin \theta-\sinh \rho)\left(1-\left(\frac{\cos \theta}{\sin \chi \cos \theta_{0}}\right)^{2}\right)
$$

is independent of $\gamma$. The $S_{W Z}$ term also is independent of $\gamma$.

We finally only consider the $S_{\theta}$ term

$$
\begin{aligned}
S_{\theta} & =-\theta_{0}^{\prime} \int d \psi d \sigma d \varphi P_{\theta}=-T_{D 3} \theta_{0}^{\prime} \int d \psi d \sigma d \varphi \frac{\delta\left(\mathcal{L}_{D B I}+S_{W Z}\right)}{\delta \theta^{\prime}} \\
& =-T_{D 3} \theta_{0}^{\prime} \int d \psi d \sigma d \varphi\left(\frac{i \sin \chi \sinh \sigma \theta^{\prime}\left(\cos ^{2} \chi \sinh ^{2} \rho+\sin ^{2} \theta\right)}{\cos \chi(A \cosh \rho \sin \theta-\sinh \rho)}+\frac{\delta S_{W Z}}{\delta \theta^{\prime}}\right) .
\end{aligned}
$$

Where $P_{\theta}$ is the conjugate momentum of $\theta$. The $S_{\theta}$ is independent of $\gamma$ from above expression.

From the above analysis, we conclude that the total on-shell D3-brane action of $1 / 4$ BPS Wilson-'t Hooft loop is independent the $\gamma$. This is to say that we can let the $\gamma=0$. As we know that the $\gamma=0$ corresponds to the Wilson loop case. We have the relation

$$
S_{\theta_{0}}^{W H}(C, \gamma)=S_{\theta_{0}}^{W H}(C, 0)=S_{\theta_{0}}^{\text {Wilsonloop }}(C) .
$$

We can use the Wilson loop result to obtain the Wilson-'t Hooft loop expectation value, but the $C$ has different physical meaning.

The expectation value of $1 / 4$ BPS Wilson loop has been calculated in [32]. The D3brane solutions (3.37), (3.38) and (3.35) are unphysical in Lorentzian space. So we analytically continue those solutions to Euclidean signature by taking the Wick rotation

$$
\chi=i u, \quad \sigma=i \vartheta .
$$

After this Wick rotation, the Euclidean version of metric (3.6) is

$$
\frac{d s^{2}}{L^{2}}=d u^{2}+\cosh ^{2} u\left(d \rho^{2}+\sinh ^{2} \rho d \psi^{2}\right)+\sinh ^{2} u\left(d \vartheta^{2}+\sin ^{2} \vartheta d \varphi^{2}\right)+d \theta^{2}+\sin ^{2} \theta d \phi^{2} .
$$

The solutions (3.37) and (3.38) become

$$
\begin{aligned}
\sinh u \cos \theta & =c, \\
\cosh u\left(\cosh \rho \cos \theta \sin \theta_{0}-\sinh \rho \sin \theta \cos \theta_{0}\right) & =d .
\end{aligned}
$$

The solution smooth at $\rho=0$ only for

$$
d= \pm \sin \theta_{0} \sqrt{1+c^{2}} .
$$

From this, we can solved $\rho$ as a function $\theta$

$$
\sinh \rho=\operatorname{sign}\left(\theta_{0}-\theta\right) \frac{\sin \theta \sin \theta_{0}\left(\sqrt{1+c^{2}} \cos \theta_{0}+\cos \theta \sqrt{1+\frac{c^{2} \cos ^{2} \theta_{0}}{\cos ^{2} \theta}}\right.}{\cosh u\left(\cos ^{2} \theta-\cos ^{2} \theta_{0}\right)} .
$$


Using this expression, the solutions can be written as function of $\theta$ instead of $\rho$. The world-volume is parameterized by $\{\theta, \psi, \vartheta, \varphi\}$. The $\rho=\rho(\theta)$ and $u=u(\theta)$ are given by the solutions above. The 1/4 BPS Wilson loop has been discussed in [32] in this coordinates. The total D3-brane action of the 1/4 BPS Wilson loop is given by

$$
S_{\theta_{0}}^{\text {Wilsonloop }}(c)=-2 N\left(c \sqrt{1+c^{2}}+\operatorname{arcsinh} c\right) .
$$

From the relation (3.47), we find

$$
S_{\theta_{0}}^{W H}(c, \gamma)=-2 N\left(c \sqrt{1+c^{2}}+\operatorname{arcsinh} c\right) .
$$

So the expectation value of $1 / 4$ BPS circular Wilson-'t Hooft loop is

$$
\left\langle W_{\theta_{0}}^{W H}(c, \gamma)\right\rangle=\exp \left[2 N\left(c \sqrt{1+c^{2}}+\operatorname{arcsinh} c\right)\right] .
$$

Although the expectation value of $1 / 4$ BPS Circular Wilson-'t Hooft loop has the same expression as Wilson loop, the $c$ has different physical meaning.

\subsubsection{The physical meaning of $c$}

The D3-brane DBI action with electric and magnetic field on its world-volume is given by

$$
S_{D B I}^{E}=2 N \int d \theta d \vartheta \sqrt{\left(\cosh ^{2} u \rho^{\prime 2}+u^{\prime 2}+1\right)\left(\cosh ^{2} u \sinh ^{2} \rho+\sin ^{2} \theta\right)+F_{\theta \psi}^{2}} \sqrt{\sinh ^{4} u \sin ^{2} \vartheta+F_{\vartheta \varphi}^{2}} .
$$

Where' stands for the derivative with respect to $\theta$. The momentum conjugate to the gauge field $A_{\psi}$

$$
\Pi=-i \frac{2 \pi \alpha^{\prime}}{L^{2}} T_{D 3} \int d \vartheta d \varphi \frac{\delta \mathcal{L}_{D B I}^{E}}{\delta F_{\theta \psi}}= \pm \frac{4 N}{\sqrt{\lambda}}\left|\frac{c}{\cos \theta_{0}}\right| \cos \gamma= \pm k .
$$

The integer charge $k$ corresponds to the number of coincident $\mathrm{F} 1$ stings.

The magnetic charge $\mathrm{m}$ is

$$
m=\frac{1}{2 \pi} \frac{L^{2}}{2 \pi \alpha^{\prime}} \int d \vartheta d \varphi F_{\vartheta \varphi}=\frac{\sqrt{\lambda}}{\pi} \frac{c}{\cos \theta_{0}} \sin \gamma
$$

The integer magnetic charge $m$ is the number of D1-branes immersed in the D3-branes. From the above two expressions, we find

$$
c^{2}=\cos ^{2} \theta_{0}\left(\frac{k^{2} \lambda}{16 N^{2}}+\frac{(m \pi)^{2}}{\lambda}\right)
$$

Using the dual coupling constant $\tilde{\lambda}=\frac{16 \pi^{2} N^{2}}{\lambda}, c$ can be written as

$$
c^{2}=\cos ^{2} \theta_{0}\left[\frac{k^{2} \lambda}{(4 N)^{2}}+\frac{m^{2} \tilde{\lambda}}{(4 N)^{2}}\right] .
$$

Without considering the axion field, the expression does not manifest the $\mathrm{SL}(2, Z)$ symmetry. 


\subsubsection{The $\mathrm{SL}(2, Z)$ symmetry}

To restore the full $\mathrm{SL}(2, Z)$ duality, we must consider the effect of a nonzero axion field $C_{0}$. For a non-zero constant axion background, the D3-brane action has additional WessZumino term

$$
S_{\text {axion }}=\mu_{3}(2 \pi \alpha)^{2} \int C_{0} F_{\rho \psi} F_{\sigma \varphi} .
$$

For the nonvanishing axion field, the supersymmetry analysis still holds because the kappa projection operator dosen't involve the any RR filed. All the calculations are the same as the zero axion case with a replacement

$$
k \rightarrow k+m C_{0} .
$$

From the AdS/CFT duality, the axion in the bulk could be identified with the $\theta$ parameter in the Yang-Mills theory

$$
C_{0}=\frac{\theta}{2 \pi} .
$$

In the nonvanishing axion background $C_{0}$, the expectation value of $1 / 4 \mathrm{BPS}$ circular Wilson-'t Hooft loop becomes

$$
\left\langle W_{\theta_{0}}^{W H}(c, \gamma)\right\rangle=\exp \left[2 N\left(c \sqrt{1+c^{2}}+\operatorname{arcsinh} c\right)\right]
$$

with

$$
\begin{aligned}
c^{2} & =\cos ^{2} \theta_{0}\left[\left(k+\frac{m \theta}{2 \pi}\right)^{2} \frac{\lambda}{(4 N)^{2}}+\frac{m^{2} \tilde{\lambda}}{(4 N)^{2}}\right] \\
& =\cos ^{2} \theta_{0} \frac{\pi}{4 N} \frac{|k+m \tau|^{2}}{I m \tau} .
\end{aligned}
$$

This is invariant under the $\mathrm{SL}(2, Z)$ duality with $S$ and $T$ transformation

$$
\begin{aligned}
S: \tau \rightarrow-\frac{1}{\tau} & (n, m) \rightarrow(-m, n) \\
T: \tau \rightarrow \tau+1 & (n, m) \rightarrow(n+m, m) .
\end{aligned}
$$

\section{Conclusions and discussions}

In this paper, we investigate the $1 / 4$ BPS Wilson-'t Hooft loops in $\mathcal{N}=4$ supersymmetric Yang-Mills theory. We use the bulk D3-brane solutions with both electric and magnetic charges to describe some of $1 / 4$ BPS Wilson-'t Hooft loops. We consider 1/4 BPS Wilson't Hooft loops with both the $F 1$ 's and $D 1$ 's in symmetric representation. Similar to the $F 1$ 's, this can be studed by using the D3-branes configuration. We calculate the conserved charges for straight $1 / 4$ BPS Wilson-'t Hooft loops and expectation value for circular $1 / 4$ BPS Wilson-'t Hooft loops.

In our paper, we mainly discuss the $1 / 4$ BPS Wilson-'t Hooft loops in symmetry representation. It would be interesting to discuss the general representation of $1 / 4$ BPS Wilson-'t Hooft loops. Similar to the case of Wilson loops, another interesting problem is the 1/4 BPS Wilson surface in six-dimensional field theory in the framework of $A d S_{7} / C F T_{6}$ correspondence $[1,44-53]$ using the $\kappa$ symmetry. 


\section{Acknowledgments}

This work is supported by the NSF of China Grant No. 11305131 and Research Start-up Foundation for Talents of Northwest A\&F University of China Grant No. Z111021106, Z111021307.

Open Access. This article is distributed under the terms of the Creative Commons Attribution License (CC-BY 4.0), which permits any use, distribution and reproduction in any medium, provided the original author(s) and source are credited.

\section{References}

[1] J.M. Maldacena, The large- $N$ limit of superconformal field theories and supergravity, Int. J. Theor. Phys. 38 (1999) 1113 [hep-th/9711200] [INSPIRE].

[2] S.S. Gubser, I.R. Klebanov and A.M. Polyakov, Gauge theory correlators from noncritical string theory, Phys. Lett. B 428 (1998) 105 [hep-th/9802109] [INSPIRE].

[3] E. Witten, Anti-de Sitter space and holography, Adv. Theor. Math. Phys. 2 (1998) 253 [hep-th/9802150] [INSPIRE].

[4] O. Aharony, S.S. Gubser, J.M. Maldacena, H. Ooguri and Y. Oz, Large-N field theories, string theory and gravity, Phys. Rept. 323 (2000) 183 [hep-th/9905111] [INSPIRE].

[5] S.-J. Rey and J.-T. Yee, Macroscopic strings as heavy quarks in large- $N$ gauge theory and anti-de Sitter supergravity, Eur. Phys. J. C 22 (2001) 379 [hep-th/9803001] [INSPIRE].

[6] J.M. Maldacena, Wilson loops in large-N field theories, Phys. Rev. Lett. 80 (1998) 4859 [hep-th/9803002] [INSPIRE].

[7] N. Drukker, D.J. Gross and H. Ooguri, Wilson loops and minimal surfaces, Phys. Rev. D 60 (1999) 125006 [hep-th/9904191] [INSPIRE].

[8] J.K. Erickson, G.W. Semenoff and K. Zarembo, Wilson loops in $N=4$ supersymmetric Yang-Mills theory, Nucl. Phys. B 582 (2000) 155 [hep-th/0003055] [INSPIRE].

[9] N. Drukker and B. Fiol, All-genus calculation of Wilson loops using D-branes, JHEP 02 (2005) 010 [hep-th/0501109] [INSPIRE].

[10] S. Yamaguchi, Wilson loops of anti-symmetric representation and D5-branes, JHEP 05 (2006) 037 [hep-th/0603208] [INSPIRE].

[11] J. Gomis and F. Passerini, Holographic Wilson loops, JHEP 08 (2006) 074 [hep-th/0604007] [INSPIRE].

[12] J. Gomis and F. Passerini, Wilson loops as D3-branes, JHEP 01 (2007) 097 [hep-th/0612022] [INSPIRE].

[13] N. Drukker and D.J. Gross, An exact prediction of $N=4$ SUSYM theory for string theory, J. Math. Phys. 42 (2001) 2896 [hep-th/0010274] [INSPIRE].

[14] G. Akemann and P.H. Damgaard, Wilson loops in $N=4$ supersymmetric Yang-Mills theory from random matrix theory, Phys. Lett. B 513 (2001) 179 [Erratum ibid. B 524 (2002) 400] [hep-th/0101225] [inSPIRE].

[15] R.C. Myers, Dielectric branes, JHEP 12 (1999) 022 [hep-th/9910053] [InSPIRE]. 
[16] D. Rodriguez-Gomez, Computing Wilson lines with dielectric branes, Nucl. Phys. B 752 (2006) 316 [hep-th/0604031] [INSPIRE].

[17] K. Okuyama and G.W. Semenoff, Wilson loops in $N=4$ SYM and fermion droplets, JHEP 06 (2006) 057 [hep-th/0604209] [INSPIRE].

[18] S. Yamaguchi, Bubbling geometries for half BPS Wilson lines, Int. J. Mod. Phys. A 22 (2007) 1353 [hep-th/0601089] [inSPIRE].

[19] O. Lunin, On gravitational description of Wilson lines, JHEP 06 (2006) 026 [hep-th/0604133] [INSPIRE].

[20] E. D'Hoker, J. Estes and M. Gutperle, Gravity duals of half-BPS Wilson loops, JHEP 06 (2007) 063 [arXiv:0705.1004] [INSPIRE].

[21] S.A. Hartnoll and S.P. Kumar, Higher rank Wilson loops from a matrix model, JHEP 08 (2006) 026 [hep-th/0605027] [INSPIRE].

[22] A. Faraggi, J.T. Liu, L.A. Pando Zayas and G. Zhang, One-loop structure of higher rank Wilson loops in AdS/CFT, Phys. Lett. B 740 (2015) 218 [arXiv: 1409.3187] [INSPIRE].

[23] M.T. Grisaru, R.C. Myers and O. Tafjord, SUSY and goliath, JHEP 08 (2000) 040 [hep-th/0008015] [INSPIRE].

[24] A. Hashimoto, S. Hirano and N. Itzhaki, Large branes in AdS and their field theory dual, JHEP 08 (2000) 051 [hep-th/0008016] [INSPIRE].

[25] J. McGreevy, L. Susskind and N. Toumbas, Invasion of the giant gravitons from Anti-de Sitter space, JHEP 06 (2000) 008 [hep-th/0003075] [INSPIRE].

[26] H. Lin, O. Lunin and J.M. Maldacena, Bubbling AdS space and 1/2 BPS geometries, JHEP 10 (2004) 025 [hep-th/0409174] [INSPIRE].

[27] A. Kapustin, Wilson-'t Hooft operators in four-dimensional gauge theories and S-duality, Phys. Rev. D 74 (2006) 025005 [hep-th/0501015] [INSPIRE].

[28] F. Pucci, More on 't Hooft loops in $N=4$ SYM, JHEP 11 (2012) 161 [arXiv:1207.6627] [INSPIRE].

[29] E. Witten, Bound states of strings and p-branes, Nucl. Phys. B 460 (1996) 335 [hep-th/9510135] [INSPIRE].

[30] B. Chen and W. He, On 1/2-BPS Wilson-'t Hooft loops, Phys. Rev. D 74 (2006) 126008 [hep-th/0607024] [INSPIRE].

[31] N. Drukker and S. Kawamoto, Small deformations of supersymmetric Wilson loops and open spin-chains, JHEP 07 (2006) 024 [hep-th/0604124] [INSPIRE].

[32] N. Drukker, S. Giombi, R. Ricci and D. Trancanelli, On the D3-brane description of some 1/4 BPS Wilson loops, JHEP 04 (2007) 008 [hep-th/0612168] [INSPIRE].

[33] E. Bergshoeff and P.K. Townsend, Super D-branes, Nucl. Phys. B 490 (1997) 145 [hep-th/9611173] [INSPIRE].

[34] E. Bergshoeff, R. Kallosh, T. Ortín and G. Papadopoulos, Kappa symmetry, supersymmetry and intersecting branes, Nucl. Phys. B 502 (1997) 149 [hep-th/9705040] [INSPIRE].

[35] K. Skenderis and M. Taylor, Branes in AdS and pp wave space-times, JHEP 06 (2002) 025 [hep-th/0204054] [INSPIRE]. 
[36] Y. Imamura, Supersymmetries and BPS configurations on Anti-de Sitter space, Nucl. Phys. B 537 (1999) 184 [hep-th/9807179] [INSPIRE].

[37] M. Cederwall, A. von Gussich, B.E.W. Nilsson and A. Westerberg, The Dirichlet super three-brane in ten-dimensional type IIB supergravity, Nucl. Phys. B 490 (1997) 163 [hep-th/9610148] [INSPIRE].

[38] M. Aganagic, C. Popescu and J.H. Schwarz, D-brane actions with local kappa symmetry, Phys. Lett. B 393 (1997) 311 [hep-th/9610249] [INSPIRE].

[39] M. Cederwall, A. von Gussich, B.E.W. Nilsson, P. Sundell and A. Westerberg, The Dirichlet super p-branes in ten-dimensional type IIA and IIB supergravity, Nucl. Phys. B 490 (1997) 179 [hep-th/9611159] [INSPIRE].

[40] M. Aganagic, C. Popescu and J.H. Schwarz, Gauge invariant and gauge fixed D-brane actions, Nucl. Phys. B 495 (1997) 99 [hep-th/9612080] [INSPIRE].

[41] N. Drukker and B. Fiol, On the integrability of Wilson loops in $A d S_{5} \times S^{5}$ : some periodic ansätze, JHEP 01 (2006) 056 [hep-th/0506058] [INSPIRE].

[42] K. Zarembo, Supersymmetric Wilson loops, Nucl. Phys. B 643 (2002) 157 [hep-th/0205160] [INSPIRE].

[43] N. Drukker, 1/4 BPS circular loops, unstable world-sheet instantons and the matrix model, JHEP 09 (2006) 004 [hep-th/0605151] [INSPIRE].

[44] O. Aharony, M. Berkooz, S. Kachru, N. Seiberg and E. Silverstein, Matrix description of interacting theories in six-dimensions, Adv. Theor. Math. Phys. 1 (1998) 148 [hep-th/9707079] [INSPIRE].

[45] O.J. Ganor, Six-dimensional tensionless strings in the large- $N$ limit, Nucl. Phys. B 489 (1997) 95 [hep-th/9605201] [INSPIRE].

[46] O. Aharony, M. Berkooz and N. Seiberg, Light cone description of $(2,0)$ superconformal theories in six-dimensions, Adv. Theor. Math. Phys. 2 (1998) 119 [hep-th/9712117] [INSPIRE].

[47] A.J. Nurmagambetov and I.Y. Park, On the $M 5$ and the $A d S_{7} / C F T_{6}$ correspondence, Phys. Lett. B 524 (2002) 185 [hep-th/0110192] [INSPIRE].

[48] B. Chen, W. He, J.-B. Wu and L. Zhang, M5-branes and Wilson surfaces, JHEP 08 (2007) 067 [arXiv:0707.3978] [INSPIRE].

[49] B. Chen, C.-Y. Liu and J.-B. Wu, Operator product expansion of Wilson surfaces from M5-branes, JHEP 01 (2008) 007 [arXiv:0711.2194] [INSPIRE].

[50] O. Aharony, O. Bergman, D.L. Jafferis and J. Maldacena, $N=6$ superconformal Chern-Simons-matter theories, M2-branes and their gravity duals, JHEP 10 (2008) 091 [arXiv:0806.1218] [INSPIRE].

[51] B. Chen and J.-B. Wu, Wilson-Polyakov surfaces and M-theory branes, JHEP 05 (2008) 046 [arXiv: 0802.2173] [INSPIRE].

[52] C.-Y. Liu, Wilson surface correlator in the $A d S_{7} / C F T_{6}$ correspondence, JHEP 07 (2013) 009 [INSPIRE].

[53] H. Mori and S. Yamaguchi, M5-branes and Wilson surfaces in $A d S_{7} / C F T_{6}$ correspondence, Phys. Rev. D 90 (2014) 026005 [arXiv:1404.0930] [InSPIRE]. 